\title{
Usefulness of endoscopic nasobiliary drainage-guided ERCP in patients with surgically altered anatomy (with video) $\square$
}

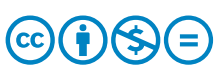

Yuichi Takano, Tetsushi Azami, Fumitaka Niiya, Takahiro Kobayashi, Naotaka Maruoka, Masatsugu Nagahama

\author{
Authors \\ Institution \\ Division of Gastroenterology, Department of Internal \\ Medicine, Showa University Fujigaoka Hospital, Yokohama, \\ Kanagawa, Japan \\ submitted 17.12.2019 \\ accepted after revision $\quad 2.3 .2020$ \\ Bibliography \\ DOI https://doi.org/10.1055/a-1149-1619 | \\ Endoscopy International Open 2020; 08: E805-E808 \\ (c) Georg Thieme Verlag KG Stuttgart · New York \\ eISSN 2196-9736 \\ Corresponding author \\ Yuichi Takano, 1-30 Fujigaoka, Aoba-ku, Yokohama-shi, \\ Kanagawa 227-8501, Japan \\ Fax: +81-45-9731019 \\ yuichitakano1028@yahoo.co.jp
}

\section{ABSTRACT}

Background and study aims Endoscopic retrograde cholangiopancreatography (ERCP) in patients with surgically altered anatomy is one of the most challenging endoscopic procedures. Although single- or double-balloon endoscopes have been widely used, reaching the papilla of Vater (hepaticojejunostomy/pancreaticojejunostomy site) is often difficult. For patients in whom treatment cannot be completed in a single session, we placed endoscopic nasobiliary drainage (ENBD) at the end of the procedure; in the second session, the scope was inserted following ENBD placement.

Patients and methods Three patients with surgically altered anatomy and who underwent ENBD-guided ERCP were retrospectively examined using the medical records. Results There were two men and one woman, with an average age of 75 years. The surgical procedure were distal gastrectomy and Roux-en-Y reconstruction in all patients. The diagnosis were choledocholithiasis in two and bile duct stricture in one. Average time to reach the papilla was 50 minutes (range, 21-102) for the first ERCP and was shortened to 11 minutes (range, 5-17) for the second session under an indwelling ENBD. Treatment was successful in all patients without complications.

Conclusion ENBD-guided ERCP in patients with surgically altered anatomy was a useful method that facilitated scope insertion and shortened the procedure time.

\section{Introduction}

Endoscopic retrograde cholangiopancreatography (ERCP) in patients with surgically altered anatomy is one of the most difficult endoscopic procedures [1]. Although single- or doubleballoon endoscopes have been widely used, reaching the papilla of Vater is often difficult [2-5]. Therefore, completing the treatment in a single session is desirable as performing a second session would entail restarting from scope insertion. However, in clinical practice, completion of treatment in a single session is difficult in some cases, such as in cases of stacked stones. For such cases, we have been placing 6-Fr endoscopic nasobiliary drainage (ENBD) at the end of the procedure. Dur- ing the second session, we attempt to insert the scope following ENBD placement.

\section{Case reports}

Three consecutive patients who underwent ENBD-guided ERCP from 2018 to 2019 were retrospectively reviewed ( $\triangleright$ Table $\mathbf{1}$ ). There were two men and one woman, with an average age of 75 years. The surgical procedures were distal gastrectomy and Roux-en-Y reconstruction in all patients. Indications for a second ERCP were residual stones in two and internal drainage in one. Average time to the second procedure was 3 days (with no analgesia needed). 
Table 1 Clinical characteristics of three patients.

\begin{tabular}{|l|l|l|l|}
\hline Case & $\mathbf{1}$ & $\mathbf{2}$ & $\mathbf{3}$ \\
\hline Age & 81 & 73 & 70 \\
\hline Sex & Female & Male & Male \\
\hline Diagnosis & Choledocholithiasis & Bile duct stricture(malignant) & Choledocholithiasis \\
\hline Gastrointestinal anatomy & Distal gastrectomy, Roux-en-Y & Distal gastrectomy, Roux-en-Y & Distal gastrectomy, Roux-en-Y \\
\hline Indications for a second ERCP & Residual stones & Internal drainage & Residual stones \\
\hline Time to second procedure(days) & 3 & 4 & 3 \\
\hline $\begin{array}{l}\text { Time to reach the papilla during the first ERCP } \\
\text { (min) }\end{array}$ & 21 & 102 & 27 \\
\hline $\begin{array}{l}\text { Time to reach the papilla during the second } \\
\text { ERCP with ENBD placement (min) }\end{array}$ & 5 & 17 & 11 \\
\hline $\begin{array}{l}\text { Complications } \\
\text { ERCP, endoscopic retrograde cholangiopancreatography; ENBD, endoscopic nasobiliary drainage. }\end{array}$ \\
\hline
\end{tabular}


- Fig. 1 In Case 1, Aa 81-year-old woman with a history of distal gastrectomy and Roux-en-Y reconstruction was admitted for common bile duct stones and cholecystitis. ERCP using short-type single-balloon endoscope (SIF-290S, Olympus Medical Systems, Tokyo, Japan) was performed. a For the first ERCP, time to reach the papilla was 21 minutes. Cholangiography showed numerous stones in the common bile duct. $\mathbf{b}$ Complete stone extraction was impossible during the first session, and a 6-Fr pigtail-type ENBD was placed at the end of the procedure. c During the second session with the ENBD in place, the scope was able to reach the papilla in 5 minutes. $\mathbf{d}$ The anastomosis was easily recognized under an indwelling ENBD. e The ampulla of Vater with the ENBD in place. 


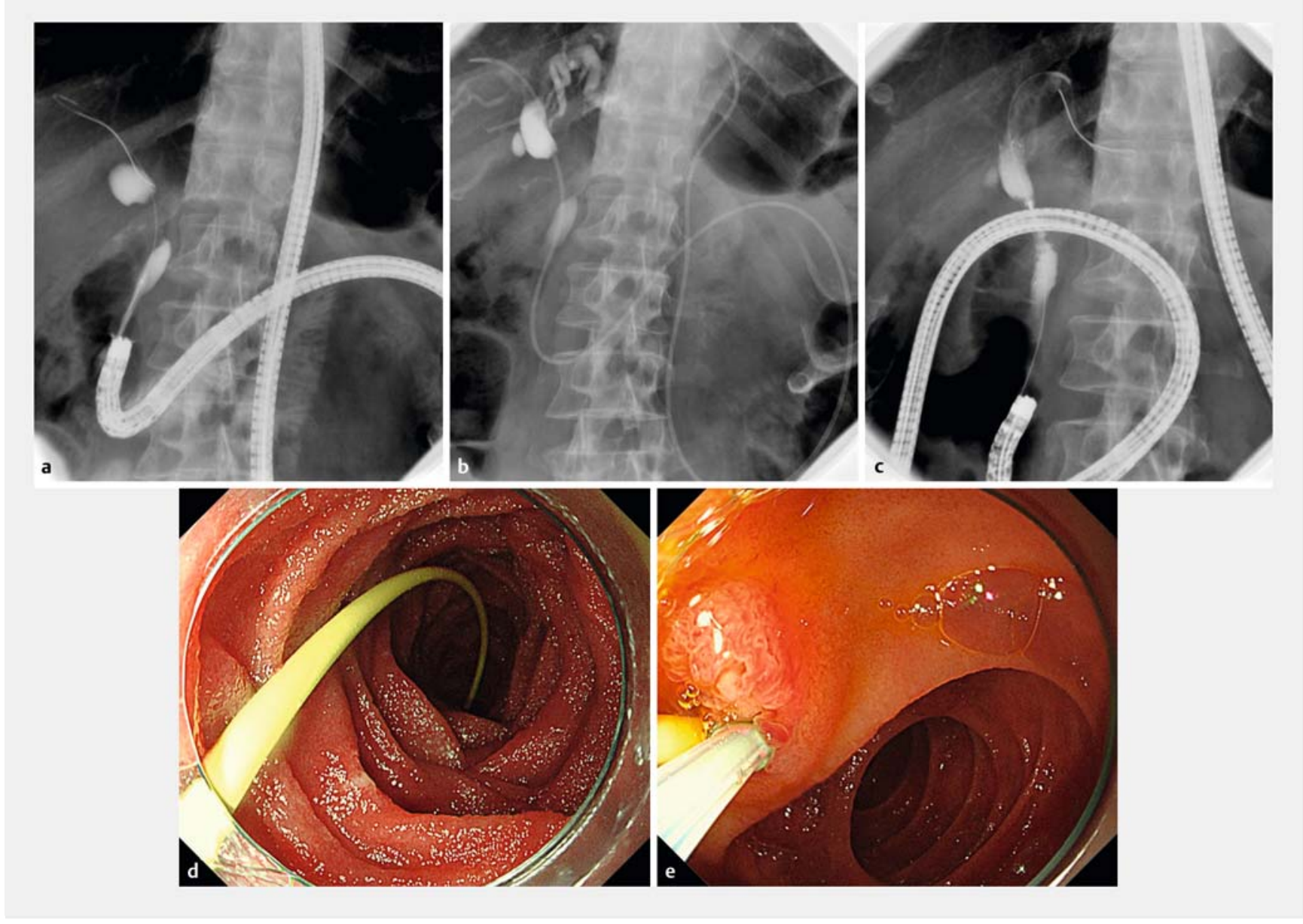

- Fig. 2 In Case 2, a 73-year-old man with a history of distal gastrectomy Roux-en-Y reconstruction for gastric cancer. The patient was transported from a previous doctor owing to obstructive jaundice. a First ERCP was performed using short-type single-balloon endoscope. Owing to a high degree of adhesion, it took to 102 minutes to reach the papilla. Cholangiography shows stricture in the middle bile duct. b A 6-Fr ENBD was placed for reducing jaundice. $c$ A second ERCP was performed for internal drainage. After insertion of the scope along the ENBD, it reached the papilla in 17 minutes. A metallic stent was placed, and jaundice improved. $\mathbf{d}$ The scope could be inserted smoothly using ENBD as a guide. e Advancement of the catheter along the ENBD successfully allowed deep cannulation of the bile duct.

Average time to reach the papilla during the first ERCP was 50 minutes (range, 21-102) and was shortened to 11 minutes (range, 5-17) during the second ERCP. Treatment was successful in all patients. There were no complications associated with

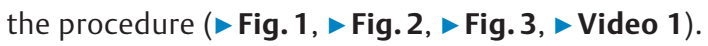

With this procedure, the scope can be inserted smoothly using ENBD as a guide. In patients with surgically altered anatomy who require a second ERCP, this technique could be an effective option. However, the disadvantages of ENBD placement include patient discomfort, aspiration pneumonia (caused by saliva entering the lungs due to dysphagia), and risk of self-extraction. Therefore, this procedure may not be feasible in cases with dementia or impaired swallowing function.

\section{Competing interests}




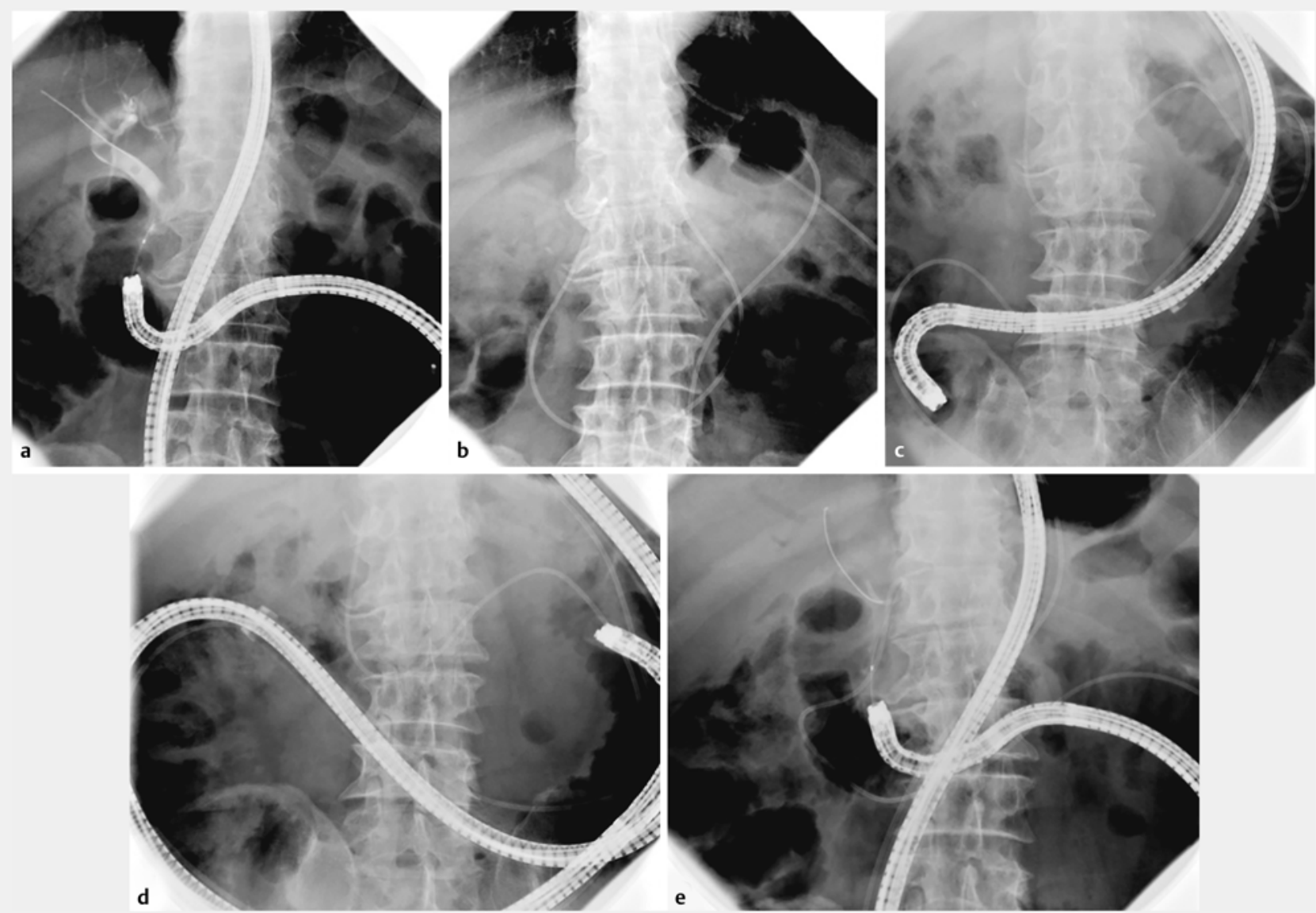

- Fig. 3 In Case 3, 70-year-old man with a history of distal gastrectomy Roux-en-Y reconstruction was admitted for common bile duct stones and cholangitis. a For the first ERCP, time to reach the papilla was 27 minutes. Cholangiography shows multiple stones in the common bile duct. b Complete stone extraction was impossible during the first session, and a 6-Fr pigtail-type ENBD was placed. c, d, e Three days later, a second ERCP was performed. The scope was inserted with the ENBD in place. The time to reach the papilla was shortened to 11 minutes.

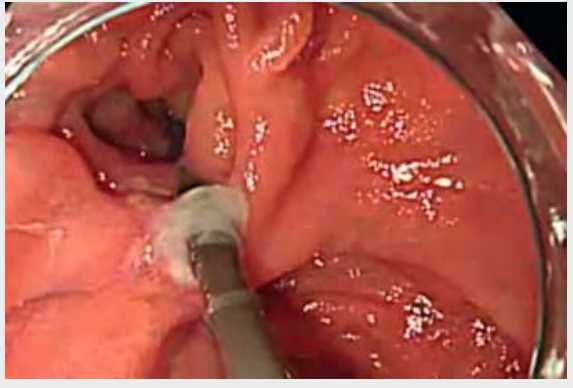

Video 1 In Case 1, a single-balloon endoscope was inserted to follow the ENBD. After confirming the anastomosis, smooth scope insertion into the afferent loop was possible, and it reached the papilla in 5 minutes.

\section{References}

[1] Wright BE, Cass OW, Freeman ML. ERCP in patients with long-limb Roux-en-Y gastrojejunostomy and intact papilla. Gastrointest Endosc 2002; 56: 225-232

[2] Saleem A, Baron TH, Gostout C] et al. Endoscopic retrograde cholangiopancreatography using a single-balloon enteroscope in patients with altered Roux-en-Y anatomy. Endoscopy 2010; 42: 656-660

[3] Shimatani M, Takaoka M, Tokuhara M et al. Review of diagnostic and therapeutic endoscopic retrograde cholangiopancreatography using several endoscopic methods in patients with surgically altered gastrointestinal anatomy. World J Gastrointest Endosc 2015; 7: 617-627

[4] Ishii K, Itoi T, Tonozuka R et al. Balloon enteroscopy-assisted ERCP in patients with Roux-en-Y gastrectomy and intact papillae (with videos). Gastrointest Endosc 2016; 83: 377-386

[5] Katanuma A, Isayama H. Current status of endoscopic retrograde cholangiopancreatography in patients with surgically altered anatomy in Japan: questionnaire survey and important discussion points at Endoscopic Forum Japan 2013. Dig Endosc 2014; 26: 109-115 